\section{Características clínico- epidemiológicas no carcinoma espinocelular de boca e orofaringe}

\author{
Rogério A. Dedivitis' ${ }^{1}$, Cristiane M. França², \\ Ana Claudia B. Mafra³, Fernanda T. Guimarães ${ }^{3}$, \\ André V. Guimarães
}

\section{Resumo / Summary}

câncer de boca e orofaringe é de comportamento agressivo e, no Brasil, a incidência é considerada uma das mais altas do mundo, sendo o mais comum da região de cabeça e pescoço. Objetivo: 0 objetivo deste trabalho é analisar os aspectos clínico-epidemiológicos dos pacientes e a evolução da doença. Forma de estudo: Clínico retrospectivo. Casuística e Método: Foram arrolados 43 casos de carcinoma espinocelular (CEC) de boca e 25 de orofaringe do Serviço de Cirurgia de Cabeça e Pescoço da Santa Casa de Misericórdia de Santos e do Hospital Ana Costa entre os anos de 1997 a 2000. Informações sobre idade, sexo, profissão, raça, hábitos de tabagismo e etilismo, uso de prótese dentária, origem do encaminhamento do paciente ao tratamento, localização da lesão, estadiamento clínico, grau de diferenciação histológica, tratamento, sobrevida e presença de segundo tumor primário foram analisados estatisticamente pelo Teste Exato de Fischer. Resultados: Dos pacientes com CEC em boca, a relação de incidência masculino-feminino foi de 3,35:1, a idade variou de 46 a 91 anos (mediana de 62), 90,7\% eram caucasianos, $81 \%$ foram referenciados por profissionais médicos, $76,8 \%$ eram tabagistas, $74 \%$ etilistas, $79 \%$ não utilizavam prótese dentária. 0 sítio mais acometido foi a língua (51,1\%), 53\% ap resentaram-se nos estádios III e IV, 72,1\% eram de grau histológico II, 53\% foram tratados por cirurgia e $47 \%$ por cirurgia e radioterapia adjuvante e $9,3 \%$ apresentaram segundo tumor primário. Para a orofaringe, a relação masculino-feminino foi de 11,5:1, com idade entre 40 e 81 anos (mediana de 58 ), 92\% eram caucasianos, $92 \%$ foram encaminhados por médicos, $84 \%$ eram tabagistas, $80 \%$ etilistas, $52 \%$ não utilizavam prótese, as tonsilas palatinas foram o sítio mais acometido (76\%), 96\% estavam em estádios III e IV, $84 \%$ eram de grau II, $80 \%$ foram tratados por cirurgia associada a radioterapia, $16 \%$ a cirurgia para resgate de falha após radioterapia e $4 \%$ a cirurgia exclusiva e $8 \%$ tiveram segundo primário. Não houve relação estatisticamente significativa entre o estadiamento e os hábitos de tabagismo, etilismo e uso de prótese. Tais hábitos, a faixa etária e o grau histológico não tiveram relação significativa com o sítio do tumor. Estavam vivos e livres de doença $69,7 \%$ dos pacientes com tumor de boca e $22 \%$ de orofaringe. Conclusão: 0 médico ou dentista que dá o atendimento inicial é fundamental no reconhecimento das lesões, para que se possa estabelecer o diagnóstico precoce.

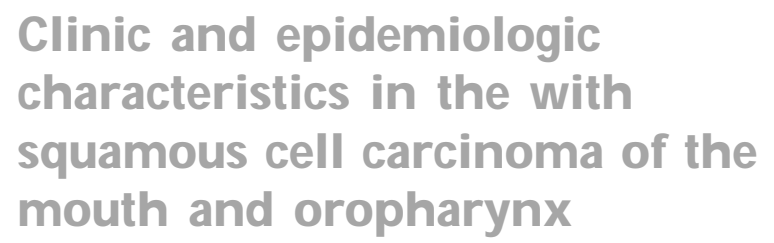

Palavras-chave: carcinoma espinocelular, câncer bucal, boca, orofaringe, epidemiologia.

Key words: squamous cell carcinoma, oral cancer, mouth, oropharynx, epidemiology. ancer of the oral cavity and oropharynx is aggressive. It is one of the commonest cancers in Brazil and may be considered as the commonest in the head and neck. Aim: The objective of this paper is to evaluate clinical and epidemiological factors and the outcome. Study design: Clinical retrospective. Material and Methods: In a descriptive retrospective study, the charts of 43 cases of oral and 25 of oropharyngeal squamous cell carcinoma (SCC) in the period 1997 2000 have been reviewed from the Departments of Head and Neck Surgery of Santa Casa de Misericórdia de Santos and Hospital Ana Costa. This was analyzed with emphasis on age, gender, profession, ethnic aspects, tobacco and alco hol use, dental prosthesis, referement origin, site of the lesion, clinical staging, histologic grade, treatment methods, survival and second cancer presence in the study group. The data were analysed by Exact Test of Fischer. Results: In the oral cavity cancer patients, a male female ratio of 3.35:1 was observed, the median age was 62 years (ranging 46 to 91 years), 90.7\% were Caucasian, $81 \%$ were referred from medical professionals, tobacco use was identified in $76.8 \%$, alcohol intake in $74 \%, 79 \%$ were not dental prosthesis users, tongue was the commonest site identified (51.1\%), 53\% were staged as III and IV clinical stages, $72.1 \%$ were moderately differentiated SCC, combined modality of treatment (surgery and adjuvant radiation therapy) was employed in $47 \%$ and 9.3\% presented a second primary tumor. For the oropharynx, the male female ratio was 11.5:1, the median age was 58 years (ranging 40 to 81 years), 92\% were Caucasian, $92 \%$ were referred from medical professionals, exposure to tobacco and alcohol was respectively noted in $84 \%$ and $80 \%, 52 \%$ did not use dental prosthesis, the tonsils were the commonest site (76\%), 96\% were staged as III and IV, $84 \%$ had moderately differentiated SCC, 75\% underwent combined treatment (surgery and adjuvant radiation therapy) and $8 \%$ presented a second tumor elsew here. There was not significant relationship between the clinical staging and to bacco, alcohol and dental prosthesis exposure. These factors, the age and the histologic grade had no relationship with the tumor site. For the oral cavity, $69,7 \%$ were alive with no evidence of disease and for the oropharynx, $22 \%$ were under this condition. Conclusion: The professional who performs the first evaluation is important in recognizing the lesions in order to achieve early detection.

\footnotetext{
${ }^{1}$ Doutor em O torrinolaringologia e Cirurgia de Cabeça e Pescoço pela UNIFESP - Escola Paulista de Medicina. ${ }^{2}$ Doutora em Patologia Bucal pela Universidade de São Paulo.

${ }^{3}$ Ex-Aluna de Graduação do Curso de O dontologia da Universidade Metropolitana de Santos (UNIMES).

${ }^{4}$ Mestre em Cirurgia de Cabeça e Pescoço pelo Curso de Pós-Graduação em Cirurgia de Cabeça e Pescoço do Complexo Hospitalar Heliópolis, São Paulo. Instituição: Disciplina de O torrinolaringologia e Cirurgia de Cabeça e Pescoço e Disciplina de Patologia Geral e Bucal da Faculdade de O dontologia da Universidade Metropolitana de Santos "Unimes".

Endereço para Correspondência: Rogério A. Dedivitis - Rua Olinto Rodrigues Dantas, 343 conjunto 92 Santos SP11050-220.

Tel(0xx13) 3223-5550/3221-1514 - E-mail: dedivitis.hns@uol.com.br

Artigo recebido em 07 de setembro de 2003. Artigo aceito em 15 de janeiro de 2003.
} 


\section{INTRODUÇÃO}

Câncer de cabeça e pescoço é rep resentado na sua imensa maioria por neoplasias epiteliais do tipo carcinoma espinocelular (CEC) que acometem as vias aerodigestivas superiores. Trata-se de doenças neoplásicas das mais freqüentes no Brasil ${ }^{1}$. 0 tratamento destas neoplasias é complexo, de caráter multidisciplinar e multimodal. As taxas de controle local e de sobrevida aumentaram com tratamentos loco-regionais avançados nos últimos 30 anos, porém, não mudaram significativamente mais recentemente ${ }^{2}$.

0 câncer de boca e orofaringe está relacionado principalmente ao tabagismo e etilismo. Ambos acometem principalmente indivíduos do sexo masculino e acima de 50 anos. Mais de $90 \%$ dos casos constituem-se do CEC. O comportamento é bastante agressivo, apresentando metastatização cervical precoce e, com freqüência, contra-lateral, já que, sobretudo em orofaringe, os linfáticos cruzam a linha média³.

0 câncer da orofaringe tem como agravante ser oligossintomático no início, seja devido ao padrão de inervação sensitiva, dada pelo glossofaríngeo, seja devido à superfície irregular da mucosa, em especial a das tonsilas palatinas, com suas criptas, onde um pequeno carcinoma pode ficar oculto ao exame clínico, ou ainda pelo padrão de inspeção do paciente, nem sempre muito atento ${ }^{3}$.

No Brasil, a incidência do câncer bucal é considerada uma das mais altas do mundo, estando entre os 6 tipos de câncer mais comuns que acometem o sexo masculino e entre os 8 mais comuns que atingem o sexo feminino. Pode ser considerado o câncer mais comum da região de cabeça e pescoço, excluindo-se o câncer de pele.

Foram analisados prontuários dos casos de carcinoma espinocelular de boca e orofaringe do Serviço de Cirurgia de Cabeça e Pescoço do Hospital Ana Costa e do Setor de Cirurgia de Cabeça e Pescoço da Irmandade da Santa Casa de Misericórdia de Santos no período de 1997 e 2000, com o objetivo de caracterizar os pacientes submetidos a tratamento cirúrgico com e sem radioterapia, para definir os fato res mais significativos para o diagnóstico precoce.

\section{CASUÍSTICA E MÉTODO}

O projeto de pesquisa, sob o número 066/02 de 27/ 08/2202 foi aprovado pela Comissão de Ética de Pesquisa da Universidade Metropolitana de Santos, vinculado à CONEP. Os dados do trabalho foram obtidos a partir da análise dos prontuários dos Serviços de Cabeça e Pescoço da Santa Casa de Misericórdia de Santos e do Hospital Ana Costa entre os anos de 1997 a 2000. Foram arrolados 43 casos de CEC de boca e 25 de orofaringe. Além destes, foram ainda excluídos do presente estudo 7 casos de CEC de boca e 5 de orofaringe, pois os respectivos prontuários não estavam com os dados completos. Todas as cirurgias foram realizadas pelos dois autores cirurgiões. Foram também exclu- ídos pacientes cujo tratamento fosse a radioterapia, associada ou não a quimioterapia.

Informações sobre idade, sexo, profissão, raça, hábitos de tabagismo e etilismo, localização da lesão, estadiamento clínico, tratamento, grau de diferenciação histológica, sobrevida, origem do encaminhamento do paciente ao tratamento (otorrinolaringologistas, outros médicos, dentistas ou o próprio paciente) uso de prótese total e presença de segundo tumor primário foram levantados e compilados para uma ficha específica.

Posteriormente os dados foram analisados e tratados estatisticamente segundo o Teste Exato de Fischer. Para tanto, correlacionamos o estadiamento clínico aos hábitos de etilismo e de tabagismo para boca e para orofaringe; o sítio primário com o uso de prótese dentária, com a idade e com o grau histológico; o profissional que realizou o encaminhamento com o estadiamento do tumor; e a sobrevida com o estadiamento. Foram ainda caracterizadas as modalidades de tratamento empregadas.

\section{Caracterização da Amostra}

Dos pacientes com CEC em boca, 33 eram do sexo masculino ( $77 \%$ ) e 10 do sexo feminino (23\%), enquanto dos pacientes com lesão em orofaringe, havia 23 do sexo masculino (92\%) e 2 do sexo feminino (8\%) (Tabela 1).

A idade dos indivíduos com CEC de boca variou entre 46 e 91 anos (mediana de 62 anos). A faixa etária mais atingida estava entre 60 e 69 anos. Os indivíduos com CEC de orofaringe apresentaram idade entre 40 e 81 anos (mediana de 58 anos), sendo a faixa etária mais atingida entre 50 e 59 anos (Tabela 2).

\section{Tabela 1. Distribuição quanto ao sexo}

\begin{tabular}{lcccc}
\hline Sexo & Boca $(\mathbf{n})$ & $\%$ & Orofaringe $(\mathrm{n})$ & $\%$ \\
\hline Masculino & 33 & 77 & 23 & 92 \\
Feminino & 10 & 23 & 2 & 8 \\
\hline Total & 43 & 100 & 25 & 100 \\
\hline
\end{tabular}

Tabela 2. Distribuição quanto à faixa etária

\begin{tabular}{lcccc}
\hline Faixa etária & Boca $(\mathbf{n})$ & $\%$ & Orofaringe & $\%$ \\
\hline 40 a 49 & 4 & 9 & 5 & 20 \\
50 a 59 & 12 & 28 & 10 & 40 \\
60 a 69 & 20 & 47 & 8 & 32 \\
70 a 79 & 5 & 12 & 1 & 4 \\
80 a 89 & 1 & 2 & 1 & 4 \\
90 a 99 & 1 & 2 & 0 & 0 \\
\hline Total & 43 & 100 & 25 & 100 \\
\hline
\end{tabular}


Q uanto à profissão, ambos grupos eram constituídos em sua maioria por aposentados e donas de casa, que corresponderam a $65,1 \%$ dos pacientes com CEC de boca $(n=28)$ e $68 \%$ daqueles com CEC de orofaringe $(n=17)$.

No grupo com CEC de boca, apenas 4 pacientes eram não-caucasianos (9,3\%), enquanto no grupo de CEC de orofaringe, 2 eram não-caucasianos (8\%).

Os pacientes com CEC de boca foram encaminhados ao Serviço de Cirurgia de Cabeça e Pescoço predominantemente por médicos ( $n=36-81 \%$ ), seguidos por dentistas ( $n$ $=6-14 \%$ ) e dois pacientes foram por si próprios buscar tratamento (5\%). Q uanto aos pacientes com CEC de orofaringe, $92 \%$ foram encaminhados por médicos $(n=23), 4 \%$ por dentistas $(n=1)$ e $4 \%$ foram por si mesmos $(n=1)$ (Tabela 3 ).

Com relação ao tabagismo, 15 pacientes $(35 \%)$ do grupo de CEC de boca fumavam até 40 cigarros por dia e 10 indivíduos (23,2\%) não apresentavam antecedentes. Nos pacientes de CEC de orofaringe 12 pacientes (48\%) fumavam até 40 cigarros por dia e 4 pacientes (16\%) não fumavam (Tabela 4). 0 hábito do etilismo foi relatado em 32 (74\%) pacientes com CEC de boca, enquanto os demais relataram não ingerir bebida alcoólica. 0 hábito do etilismo nos pacientes com CEC de orofaringe foi relatado por 20 (80\%) indivíduos e ausência desse hábito em 5 (20\%) pacientes (Tabela 5).

Q uanto ao uso de próteses dentárias, 34 dos pacientes (79\%) com CEC de boca não as utilizavam, 8 (19\%) possuíam próteses superiores e inferiores e $1(2 \%)$ prótese superior. Dos pacientes com CEC de orofaringe, 13 (52\%) não faziam uso de prótese, 9 (36\%) usavam prótese superior e inferior e 3 (12\%) usavam prótese superior (Tabela 6).

No grupo de CEC de boca, a língua foi o sítio de maior freqüência, com 22 casos registrados (51,1\%); seguido por soalho, com 11 (25,5\%); área retromolar, com 4 (9,3\%); pilar amigdalino anterior, com 3 (6,9\%); mucosa jugal com 2 (4,6\%); e 1 lesão em rebordo alveolar (2,3\%). No grupo de CEC de orofaringe, 19 pacientes tiveram CEC em tonsila palatina (76\%), 5 em base de língua (20\%) e 1 em palato mole (4\%) (Tabela 7).

0 estadiamento do tumor no grupo de CEC de boca mostrou: $1(2,3 \%)$ carcinoma in situ; $5(11,6 \%)$ como estádio I; 14 (32,5\%) estádio II; 12 (27,9\%) casos estádio III; e 11 (25,5\%) como estádio IV. 0 grupo de CEC de orofaringe apresentou 1 (4\%) como estádio II; 10 pacientes no estádio III (40\%) e 14 (56\%) pacientes no estádio IV (Tabela 8).

Q uanto ao grau de diferenciação histológica nos pacientes de CEC de boca foram encontrados: 2 pacientes $(4,7 \%)$ com neoplasia in situ, 9 pacientes $(20,9 \%)$ com grau I (bem diferenciado), 31 pacientes $(72,1 \%)$ com grau II (moderadamente diferenciado) e 1 paciente $(2,3 \%)$ grau III (pouco diferenciado). Já nos pacientes de CEC de orofaringe encontramos: 2 pacientes (8\%) grau I (bem diferenciado), 21 pacientes (84\%) grau II (moderadamente diferenciado) e 1 paciente (8\%) grau III (pouco diferenciado) (Tabela 9).
Tabela 3. Distribuição quanto ao encaminhamento

\begin{tabular}{lcccc}
\hline Encaminhamento & Boca $(\mathrm{n})$ & $\%$ & Orofaringe $(\mathrm{n})$ & $\%$ \\
\hline Médico & 35 & 81 & 23 & 92 \\
Dentista & 6 & 14 & 1 & 4 \\
O próprio paciente & 2 & 5 & 1 & 4 \\
\hline Total & 43 & 100 & 25 & 100 \\
\hline
\end{tabular}

Tabela 4. Distribuição quanto ao hábito do tabagismo

\begin{tabular}{lcccc}
\hline $\begin{array}{l}\text { No de } \\
\text { cigarros/dia }\end{array}$ & Boca $(\mathrm{n})$ & $\%$ & Orofaringe & $\%$ \\
\hline Não fuma & 10 & 23 & 4 & 16 \\
+ & 4 & 9 & 2 & 8 \\
++ & 11 & 26 & 4 & 16 \\
+++ & 3 & 7 & 3 & 12 \\
++++ & 15 & 35 & 12 & 48 \\
\hline
\end{tabular}

Obs: Cada + equivale a dez cigarros

Tabela 5. Distribuição quanto ao hábito do etilismo

\begin{tabular}{lcccc}
\hline $\begin{array}{l}\text { Ingestão } \\
\text { de álcool }\end{array}$ & Boca $(\mathrm{n})$ & $\%$ & Orofaringe & $\%$ \\
\hline Não ingere & 11 & 26 & 5 & 20 \\
+ & 6 & 14 & 3 & 12 \\
++ & 8 & 19 & 2 & 8 \\
+++ & 2 & 5 & 0 & 0 \\
++++ & 16 & 36 & 15 & 60 \\
\hline
\end{tabular}

Obs: Cada + equivale a uma dose de bebida destilada ou uma garrafa de cerveja

Tabela 6. Distribuição quanto ao tipo de prótese

\begin{tabular}{lcccc}
\hline Tipo de prótese & Boca $(\mathbf{n})$ & $\%$ & Orofaringe $(\mathrm{n})$ & $\%$ \\
\hline Não usava & 34 & 79 & 13 & 52 \\
Superior + inferior & 8 & 19 & 9 & 36 \\
Superior & 1 & 2 & 3 & 12 \\
\hline Total & 43 & 100 & 25 & 100 \\
\hline
\end{tabular}

Tabela 7. Distribuição quanto ao sítio da lesão

\begin{tabular}{llcc}
\hline & Sítio & $\mathbf{n}$ & $\%$ \\
\hline \multirow{4}{*}{ Boca } & Língua & 22 & 51 \\
& Soalho & 11 & 26 \\
& Área retromolar & 4 & 9 \\
& Pilar anterior & 3 & 7 \\
& Mucosa jugal & 2 & 5 \\
Orofaringe & Rebordo alveolar & 1 & 2 \\
\hline & Total & 43 & 100 \\
\hline & Amígdala & 19 & 76 \\
& Base de língua & 5 & 20 \\
& Palato mole & 1 & 4 \\
\hline & Total & 25 & 100
\end{tabular}




\section{RESULTADOS}

Em relação à boca, quando se correlaciona o estadiamento clínico ao hábito do tabagismo, percebe-se que não há diferença significativa entre o grupo de fumantes e não fumantes (nível descritivo $=0,8567$ ). 0 mesmo ocorre quando correlacionamos o estádio com o hábito do etilismo, onde não existe diferença significativa entre os que bebem e os que não bebem (nível descritivo $=0,9189$ ). Quanto à orofaringe, correlacionando o estadiamento ao hábito do tabagismo, não notamos diferença significativa entre os fumantes e os não fumantes e também não há entre os do is grupos comparando-os entre si (nível descritivo $=0,9999$ ). Relacionando o estádio com o hábito de etilismo, notamos uma certa tendência dos pacientes que não bebem concentrarem-se no estádio III e os que bebem no estádio IV, contudo, sem diferença significativa entre os dois grupos (nível descritivo $=0,6917$ ).

Analisamos os sítios primários em boca e orofaringe quanto ao uso de prótese dentária e não encontramos diferença significativa entre eles (nível descritivo $=0,0749$ para boca e 0,9999 para orofaringe).

Analisamos o sítio primário em função da idade dos pacientes. Nos tumores bucais, a maior incidência é na faixa etária de 50 a 59 anos e principalmente na língua e no soalho. Não existe diferença significativa entre os dois grupos (nível descritivo $=0,2102$ ). Nos tumores de orofaringe, observamos que os tumores de tonsila palatina englobam uma faixa etária bem ampla, o mesmo não acontecendo com palato mole e base de língua. Não há diferença significativa entre tonsilas e os outros dois grupos (nível descritivo $=0,4413$ ).

Tabela 8. Distribuição das lesões quanto ao estadiamento

\begin{tabular}{lcccc}
\hline Estadiamento & Boca (n) & $\%$ & Orofaringe (n) & $\%$ \\
\hline Ca in situ & 1 & 2 & 0 & 0 \\
Estádio I & 5 & 12 & 0 & 0 \\
Estádio II & 14 & 32 & 1 & 4 \\
Estádio III & 11 & 26 & 10 & 40 \\
Estádio IV & 12 & 28 & 14 & 56 \\
\hline Total & 43 & 100 & 25 & 100 \\
\hline
\end{tabular}

Relacionamos o sítio com o grau histológico dos tumores. Nos tumores de boca, notamos, em língua e soalho, predomínio dos graus I e II. Os tumores na área retromolar classificaram-se como graus II e III. Nos tumores de orofaringe observamos somente o grau II em base de língua e palato mole. Este mesmo grau foi o que prevaleceu em tonsilas palatinas.

Relacionando o profissional que encaminhou o paciente ao tratamento e o estadiamento do tumor, foi observado que tanto nos tumores bucais quanto nos de orofaringe os médicos foram os profissionais que mais encaminharam e que os dentistas encaminharam somente $10 \%$ e este tinham estádio II ou IV. Nos tumores bucais em geral os médicos em geral $(53,5 \%)$ e os otorrinolaringologistas $(27,9 \%)$ foram os profissionais que mais encaminharam e os dentistas encaminharam somente $14 \%$ dos pacientes e estes tinham estádio II ou IV, existindo possibilidade, assim, de haver diferença significativa entre os encaminhados nos dois grupos de estadiamento. Nos tumores em orofaringe, os profissionais que mais encaminharam foram os médicos em geral $(40,0 \%)$ e os otorrinolaringologistas ( $52,0 \%)$, enquanto os dentistas só encaminharam 1 paciente (4\%). Aqui, o único caso com estádio II foi encaminhado pelo otorrinolaringologista. Não houve diferença significativa entre os estadiamentos (Tabela 10).

A associação entre cirurgia e radioterapia adjuvante pós-operatória foi executada em 20 (47\%) pacientes com CEC de boca; os demais foram submetidos a cirurgia exclusiva. Para aqueles com CEC de orofaringe, $80 \%$ foram tratados por cirurgia associada a radioterapia, $16 \%$ a cirurgia para resgate de falha após radioterapia e $4 \%$ a cirurgia exclusiva. Não houve diferença significativa entre modalidade de tratamento e sobrevida.

Tabela 9. Distribuição quanto ao grau de diferenciação histológica

\begin{tabular}{lcccc}
\hline Grau & Boca $(\mathbf{n})$ & $\%$ & Orofaringe $(\mathbf{n})$ & $\%$ \\
\hline In situ & 2 & 5 & 0 & 0 \\
I & 9 & 20 & 2 & 8 \\
II & 31 & 73 & 21 & 84 \\
III & 1 & 2 & 2 & 8 \\
\hline Total & 43 & 100 & 25 & 100 \\
\hline
\end{tabular}

Tabela 10. Distribuição dos tumores quanto ao estádio e encaminhamento

\begin{tabular}{|c|c|c|c|c|c|c|}
\hline Sítio & Estádio & Dentista & Médico não ORL & Otorrino & Paciente & Total \\
\hline \multirow[t]{3}{*}{ Boca } & I e II & $0(0,0 \%)$ & $13(65,0 \%)$ & $6(30,0 \%)$ & $1(5,0 \%)$ & $20(100,0 \%)$ \\
\hline & III e IV & $6(26,1 \%)$ & $10(43,5 \%)$ & $6(26,1 \%)$ & $1(4,3 \%)$ & $23(100,0 \%)$ \\
\hline & Total & $6(14,0 \%)$ & $23(53,5 \%)$ & $12(27,9 \%)$ & $2(4,7 \%)$ & $43(100 \%)$ \\
\hline \multirow[t]{3}{*}{ Orofaringe } & I ell & $0(0,0 \%)$ & $0(0,0 \%)$ & $1(100,0 \%)$ & $0(0,0 \%)$ & $1(100,0 \%)$ \\
\hline & II e IV & $1(4,2 \%)$ & $10(4,17 \%)$ & $12(50,0 \%)$ & $1(4,2 \%)$ & $24(100,0 \%)$ \\
\hline & Total & $1(4,0 \%)$ & $10(40,0 \%)$ & $13(52,0 \%)$ & $1(4,0 \%)$ & $25(100 \%)$ \\
\hline
\end{tabular}


O tempo mínimo de seguimento dos pacientes foi de 26 meses. A sobrevida dos pacientes com CEC de boca variou de 2 a 92 meses, enquanto os pacientes com CEC de orofaringe tiveram uma sobrevida menor, variando de 1 a 40 meses. A maioria dos pacientes com CEC de boca encontra-se assintomática, ou seja, vivos sem evidência de doença 30 pacientes $(69,7 \%)$, sendo que o inverso ocorreu no grupo de CEC de orofaringe, no qual 17 (68\%) dos indivíduos foram a óbito pela doença. Notamos que os tumores de orofaringe provocam nitidamente mais óbitos que os tumores de boca, ou seja, os pacientes com tumor de orofaringe têm 2,4 vezes mais chance de morte por câncer que os pacientes com tumor de boca. Concluímos que há diferença significativa entre os dois grupos (nível descritivo $=0,0021$ ). Q uando extratificamos a casuística em estádios in situ, I e II em um grupo e III e IV em outro, mais uma vez há diferença nos resultados de sobrevida, bem melhor no primeiro grupo (gráfico 1).

Q uatro pacientes $(9,3 \%)$ com CEC de boca tiveram segundo tumor primário, sendo um em pulmão e dois em laringe, enquanto 2 pacientes com CEC de orofaringe (8\%) tiveram segundo primário, sendo um em laringe e outro em recesso piriforme.

\section{DISCUSSÃO}

No que se refere à idade, não foi verificada a ocorrência de CEC de boca em pacientes com idade inferior a 40 anos, ao contrário dos achados de outros autores ${ }^{4,5}$. A faixa etária mais atingida corresponde à descrita pelas referências acima mencionadas, mostrando-se ser a idade preferencial dos 50 aos 70 anos. Em um estudo em 264 pacientes portadores de câncer de boca com idade inferior a 35

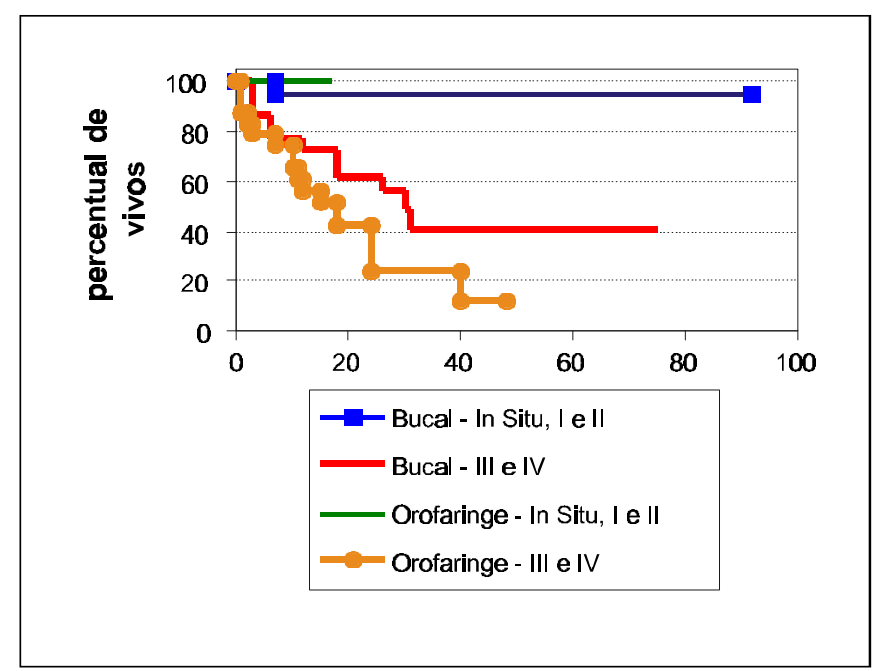

Gráfico 1. Curvas de sobrevivência para tumores de boca e de orofaringe, divididos em estádios in situ, I l II; e III e IV. anos, observou-se o hábito do tabagismo em apenas 59,4\%, indicando a necessidade de avaliação de outros fatores etiológicos ${ }^{6}$. Em nossa casuística, os pacientes tiveram significativa exposição a tabagismo e etilismo e nossos pacientes tinham idade acima dos 40 anos.

Em relação ao sexo, os dados encontrados mostramse de acordo com a literatura ${ }^{7}$, mostrando-se nos casos de CEC de boca maior incidência no sexo masculino em uma proporção de 3:1. Em nosso meio, um estudo comparou 228 pacientes do sexo feminino com 849 do masculino e encontrou, no primeiro grupo, uma faixa etária mais avançada - 60,7 anos contra 55,6 anos - , com significado estatístico ${ }^{8}$. Os autores atribuem isso à menor exposição ao tabagismo e etilismo entre as mulheres.

Em um estudo conduzido na Austrália, houve 2.173 novos casos de câncer de boca em um ano, com 400 óbitos. A maio ria dos pacientes localizava-se na faixa etária acima dos 60 anos e a prevalência do sexo masculino foi acima de 2:1. Naquele país, no entanto, mais de $50 \%$ dos casos foram de lábio ${ }^{9}$. No nosso meio, prevaleceram os casos de língua oral. Traçando um paralelo, as características da população australiana e a exposição à luz solar parecem ter um papel importante nesses números. Na nossa realidade, contudo, devemos considerar que boa parte dos tumores de lábios, sobretudo quando em estádios mais precoces, é referenciada aos cirurgiões plásticos, daí não terem uma maior representatividade em um serviço eminentemente oncológico.

Ainda quanto à localização, os achados de maior incidência em língua (50\%), seguido por soalho de boca (26\%) foram inversos a outros achados ${ }^{4}$, em que o sítio de maior freqüência foi o soalho de boca (25\%), seguido pela língua (21,87\%). Numa estratificação entre os diversos sítios acometidos em boca e orofaringe com a exposição aos principais fatores de risco, o tabagismo teve maior impacto em região jugal, alveolar, amígdalas e soalho de boca. Já o etilismo teve maior significado para região jugal e soalho de boca. Curiosamente, para língua, palato e lábio, estes fatores não foram correlacionados como de risco ${ }^{10}$.

Já o uso de próteses dentárias não foi associado a um risco maior de câncer das vias aerodigestivas superiores, mas história de úlceras orais secundárias à má adaptação foi associada ao câncer de boca e de faringe. Má higiene oral devido à baixa freqüência de escovações dentárias são também fatores de risco ${ }^{11}$. Em estudo restrospectivo sobre o risco do câncer de vias aerodigestivas superiores, o uso de próteses dentárias não foi associado a um risco aumentado, todavia história de úlceras orais secundárias à má adaptação e má higiene oral devido à baixa freqüência de escovações dentárias foram tidos como fatores de risco ${ }^{11}$. Talvez a má higiene oral permita que o carcinógeno, tabaco ou álcool, permaneça mais tempo em contato com a mucosa e, a nosso ver, faltam estudos consistentes na literatura que confirmem estas hipóteses. 
O estádio II foi o mais encontrado em nossa pesquisa (32\%). Em trabalho análogo, o estádio II foi o mais encontrado $(45,79)^{4}$. 0 grau de diferenciação histológica mais encontrado nos pacientes com CEC de boca foi o grau II, ou seja, moderadamente diferenciado (72\%), ao contrário de outros achados ${ }^{2}$ que descreveram o grau II como sendo mais freqüente em tumores de seio maxilar e soalho de boca.

Segundo o Conselho Regional de Odontologia, a cidade de Santos, no litoral paulista é uma cidade portuária com 417.983 habitantes, um sistema de saúde bem estruturado, com filosofia preventiva de atuação. Há 1.800 cirurgiões dentistas na cidade, sendo que apenas 110 são funcionários da Prefeitura. Metade da população de Santos recebe atenção odontológica pelo Sistema Unificado de Saúde. Isso gera duas realidades distintas. Por um lado, nas classes sociais mais privilegiadas a relação profissional/habitante é de $1 / 122$, enquanto a outra metade da população tem uma relação profissional/habitante de 1/1.895.

Lesões de cavidade oral, mais acessíveis ao exame clínico, são encaminhadas principalmente pelo médico em geral. Já lesões de orofaringe, não tão acessíveis, são mais encaminhadas pelo otorrinolaringologista. Nossos resultados refletem a pouca atuação do cirurgião dentista no sentido de diagnosticar e encaminhar os pacientes com câncer de boca e de orofaringe para o tratamento. Além do malefício que um diagnóstico tardio possa trazer para o paciente e para a instituição que responde pelos custos do tratamento, um outro aspecto deve ser considerado. Em uma análise retrospectiva feita nos Estados Unidos, abrangendo o período de 1.984 a 2.000, foram analisados 50 casos de veredicto de júri por má prática em câncer de boca, compreendendo um total de 21 estados americanos ${ }^{12}$. A principal razão para a interpelação judicial é a demora no diagnóstico ${ }^{12,13}$. Em uma pesquisa realizada entre dentistas no Canadá, houve 670 respostas a um questionário, sendo que $56,7 \%$ disseram estar com os conhecimentos atualizados sobre câncer de boca e orofaringe. A maioria identificou o tabagismo $(99,4 \%)$ e o etilismo $(90,4 \%)$ como fatores de risco, contudo, poucos identificaram corretamente 0 uso de alimentos com pimenta $(57 \%)$ e más condições de higiene oral $(46,3 \%)$ como não sendo fatores de risco ${ }^{14}$.

Estratégias de prevenção e educação da população, treinamento profissional mais adequado podem melhorar a abordagem destes pacientes. Estes dados indicam a necessidade de tornar-se mais eficaz o diagnóstico precoce do câncer de boca e orofaringe. A principal preocupação, a nosso ver, localiza-se na estruturação dos cursos de graduação, tanto de medicina quanto de odontologia.

\section{CONCLUSÃO}

0 presente estudo enfatiza a importância do médico ou dentista que dá o atendimento inicial realizar com eficiência o reconhecimento das lesões, para que se possa estabelecer o diagnóstico precoce.

\section{REFERÊNCIASBIBUOGRÁFICAS}

1. Franco EL, Kowalski LP, Oliveira BV, Curado MP, Pereira RN, Silva ME, Fava AS, Torloni H. Risk factors for oral cancer in Brazil: a case-control study. Int J Cancer 1989; 43:992-1000.

2. Franceschi D, Gupta R, Spiro RH, Shah JP. Improved survival in the treatment of squamous carcinoma of the oral tongue. Am J Surg 1993; 166:360-5.

3. Dedivitis RA, Guimarães AV, Souza Jr. JAL. Manual de Cirurgia de Cabeça e Pescoço. Frôntis Editorial, São Paulo; 1999, p. 64

4. Veek EB, Ribas MO, Fontanella VRC, Lorandi CSA. Estudo epidemiológico dos carcinomas espinocelulares na cavidade bucal. Ver Odonto Ciência 1992; 7:25-34.

5. Souza A, Stevaux OM, Santos GG, Marcucci G. Epidemiologia do carcinoma epidermóide da mucosa bucal - contribuição ao estudo sobre três variáveis: sexo, faixa etária e raça. Ver Odontol Unicid, 1996; 8:127-34.

6. Iype EM, Pandey M, Mathew A, Thomas G, Sebastian P, Nair MK. Oral cancer among patients under the age of 35 years. J Postgrad Med 2001; 47:171-6.

7. Gervásio OLAS, Dutra RA, Tartagli SMA, Vasconcelos WA, Barbosa AA, Aguiar MCF. Carcinoma epidermóide de boca: um estudo retrospectivo de 740 casos no Brasil. Brazil Dental J 2001; 12:57-61.

8. Carvalho MB, Lenzi J, Lehn CN, Fava AS, Amar A, Kanda JL, Walder $F$, Menezes MB, Franzi SA, Magalhães MR, Curioni $O A$, Marcel R, Szeliga S, Sobrinho JA, Rapoport A. Características clínico-epidemiológicas do carcinoma epidermóide de cavidade oral no sexo feminino. Rev Assoc Med Bras 2001; 47:208-14.

9. Sugerman PB, Savage NW. Oral cancer in Australia: 1983-1996. Austr Dent J 2002; 47:45-56.

10. Dhar PK, Rao TR, Sreekumaran N, Mohan S, Chandra S, Bhat KR, Rao K. Identification of risk factors for specific subsites within the oral and oropharyngeal region - a study of 647 cancer patients. Indian J Cancer 2000; 37:114-22.

11. Velly AM, Franco EL, Schlecht N, Pintos J, Kowalski LP, Oliveira BV, Curado MP. Relationship between dental factors and risk of upper aerodigestive tract cancer. Oral Oncology 1998; 34:284-91.

12. Lydiatt DD. Cancer of the oral cavity and medical malpractice. Laryngoscope 2002; 112:816-9.

13. Kern KA. Medicolegal analysis of the delayed diagnosis of cancer in 338 cases in the United States. Arch Surg 1994; 129:397-404.

14. Clovis JB, Horowitz AM, Poel DH. Oral and pharyngeal cancer: knowledge and opinions of dentists in British Columbia and Nova Scotia. J Can Dent Assoc 2002; 68:415-20. 